\title{
La Sentencia 2007-00012-00 del Consejo de Estado de Colombia y su conformidad con la Interpretación Prejudicial 187-IP-2015 del Tribunal Andino ${ }^{1}$
}

Judgment 2007-00012-00 by the Council of State of Colombia and its compliance with Preliminary Ruling 187-IP-2015 of the Andean Court of Justice

\section{Juan Manuel Indacochea Jauregui}

jmindacochea@gmail.com

Consultor de la Magistratura de la República del Perú en el Tribunal Andino, Ex Consultor de la Magistratura del Estado Plurinacional de Bolivia en el Tribunal Andino, Master en Derecho Internacional en la Université Paris-Ouest Nanterre La Défense, Master en Derecho de la Propiedad Intelectual en el CEIPI de l'Université de Strasbourg.

Resumen: Mediante la reciente Sentencia 2007-00012-00, el Consejo de Estado de la República de Colombia aplicó una norma comunitaria andina cuya interpretación había sido previamente solicitada al Tribunal de Justicia de la Comunidad Andina. El presente artículo analiza la citada decisión a la luz de las directrices establecidas en la interpretación prejudicial del Tribunal de Justicia de la Comunidad Andina recaída en el Proceso 187-IP-2015.

Palabras clave: Consejo de Estado (Colombia) - comunidades indígenas - expresiones culturales tradicionales - folclore - interpretación prejudicial - Tribunal Andino.

\begin{abstract}
Through the recent Judgment 2007-00012-00, the Council of State of Colombia applied an Andean Community norm related to indigenous people, whose interpretation was previously requested to the Andean Court of Justice. This paper analyzes the grounds for the Council of State's decision in light of the Andean Court of Justice Preliminary Ruling 187-IP2015.
\end{abstract}

Key words: Council of State (Colombia) - Indigenous Peoples - Traditional Cultural Expressions - Folklore - Preliminary Ruling - Andean Court of Justice.

1 Artículo enviado el 07.05.2019 y aceptado el 17.06.2019. 


\section{Introducción}

El 4 de octubre de 2018, el Consejo de Estado -máxima autoridad de la jurisdicción de lo contencioso administrativo- de la República de Colombia emitió la Sentencia 2007-00012$00^{2}$, mediante la cual determinó la irregistrabilidad del signo conformado por la denominación "Tisquesusa" y la figura del Poporo Quimbaya para distinguir servicios comprendidos en la clase 43 de la Clasificación Internacional de Niza ${ }^{3}$, declarando la nulidad de la Resolución nº 8885 de 11 de abril de 2006, expedida por la Jefe de División de Signos Distintivos de la Superintendencia de Industria y Comercio (SIC), por medio de la cual se había concedido el registro de la referida marca mixta.

El Tribunal de Justicia de la Comunidad Andina (TJCA) pronunció la interpretación prejudicial de 12 de noviembre de 2015, recaída en el Proceso 187-IP-2015, correspondiente al proceso interno 2007-00012-00, es decir, a aquel incoado por el Banco de la República de Colombia en ejercicio de la acción de nulidad relativa -ante el Consejo de Estado- en contra de la Resolución $\mathrm{N}^{\circ}$ 8883, mediante la cual se había concedido el registro de la marca "Tisquesusa" (mixta).

Mediante Sentencia 2007-00012-00, el Consejo de Estado determinó que la precitada marca era irregistrable por carecer de distintividad extrínseca toda vez que supuestamente se encontraría incursa en los tres supuestos de irregistrabilidad contemplados en el literal g) del artículo 136 de la Decisión 486, disposición normativa cuya interpretación había sido previamente solicitada -dentro del marco del mismo proceso interno- por el Consejo de Estado al TJCA en 2014.

El presente comentario de Jurisprudencia analiza los fundamentos ${ }^{4}$ de la Sentencia 200700012-00 a la luz de los criterios establecidos en la interpretación prejudicial recaída en el Proceso 187-IP-2015. Sobre la base de la redacción del literal g) del artículo $136^{5}$ de la Decisión 486 de la Comisión de la Comunidad Andina (Régimen Común sobre Propiedad

Consejo de Estado, Sentencia 2007-00012-00 de 4 de octubre de 2018 http://www.consejodeestado.gov.co/documentos/boletines/212/11001-03-24-000-2007-00012-00.pdf.

3 La marca TISQUESUSA (mixta) fue otorgada por la SIC, mediante Resolución 8883 de 11 de abril de 2006, para distinguir servicios comprendidos en la clase 43 de la Clasificación Internacional de Niza, específicamente “[...] servicios de restauración (alimentación); hospedaje temporal, cafés, bares, restaurantes, grilles y similares [...]”, en favor de Rafael Antonio Nieto Vanegas.

4 Interpretación prejudicial recaída en el Proceso 187-IP-2015 de 12 de noviembre de 2015, “Análisis del caso concreto", párr. 34 y ss.

5 Decisión 486 de la Comisión de la Comunidad Andina.

Artículo 136.- No podrán registrarse como marcas aquellos signos cuyo uso en el comercio afectara indebidamente un derecho de tercero, en particular cuando:

$[\ldots]$

g) consistan en el nombre de las comunidades indígenas, afroamericanas o locales, o las denominaciones, las palabras, letras, caracteres o signos utilizados para distinguir sus productos, servicios o la forma de procesarlos, o que constituyan la expresión de su cultura o práctica, salvo que la solicitud sea presentada por la propia comunidad o con su consentimiento expreso; y,

$[\ldots]$. 
Industrial), así como de su interpretación por parte del TJCA -y subsecuente aplicación por el Consejo de Estado-, es posible abstraer tres supuestos comprendidos en la causal de irregistrabilidad relativa establecida en la referida disposición normativa.

Teniendo en consideración lo anterior, así como el hecho de que el caso concreto atañe específicamente a las comunidades indígenas, el presente artículo se dividirá en los siguientes acápites: (I) Irregistrabilidad de signos que consistan en el nombre de comunidades indígenas; (II) Irregistrabilidad de signos utilizados para distinguir productos de comunidades indígenas; e, (III) Irregistrabilidad de signos que constituyan la expresión de la cultura o práctica de comunidades indígenas.

\section{Irregistrabilidad de signos que consistan en el nombre de comunidades indígenas}

Ante todo, es preciso aclarar que no constituye una causal de irregistrabilidad propiamente dicha ${ }^{6}$ que el signo solicitado contenga una denominación, palabra o frase en una lengua indígena reconocida en un país miembro de la Comunidad Andina (CAN).

En efecto, sería perfectamente posible registrar marcas que contengan denominaciones en lenguas indígenas (e.g., aimara, kichua o quechua ${ }^{7}$ ), salvo que se encuentren incursas en alguna causal de irregistrabilidad específica; como por ejemplo, que sean susceptibles de generar confusión conceptual o ideológica con un signo distintivo anterior ${ }^{8}$, o que consistan en el nombre de comunidades indígenas.

En ese sentido, el primer supuesto contenido en la causal de irregistrabilidad contemplada en el literal g) del artículo 136 de la Decisión 486 constituye la protección mínima que se debe conferir a las comunidades indígenas en lo concerniente al derecho de marcas. Por lo menos, éstas deben ser protegidas respecto de la utilización de su propio nombre por parte de terceras personas no autorizadas.

6 Las causales de irregistrabilidad intrínseca se encuentran comprendidas en los artículos 134 primer párrafo y 135 de la Decisión 486, mientras que aquellas de irregistrabilidad extrínseca se encuentran contenidas en el artículo 136 de la misma Decisión; dando origen las primeras a las causales de nulidad absoluta, mientras que las segundas a las causales de nulidad relativa, contempladas ambas en el primer y segundo párrafo del artículo 172, respectivamente.

7 Véase, en relación con el análisis de confundibilidad -ideológica- que involucre un signo cuyo elemento denominativo se encuentre en lengua quechua, la interpretación prejudicial del TJCA recaída en el Proceso 533-IP2015 de 26 de agosto de 2016, http://intranet.comunidadandina.org/documentos/Procesos/533IP2015.pdf.

8 Véase, además de la precitada interpretación prejudicial, las Directrices de la IPONZ (Intellectual Property Office of Nen Zealand), por ejemplo, establecen que, a efectos del examen de confundiblidad entre signos distintivos, las palabras maoríes serán tratadas de la misma manera que las marcas constituidas por palabras equivalentes en idioma inglés. Cf. IPONZ, "Absolute grounds distinctiveness. Section 18 of the Trade Marks Act 2002", 6.3 Māori words, https://www.iponz.govt.nz/about-ip/trade-marks/practice-guidelines/current/absolute-groundsdistinctiveness/\#jumpto-6 002e3-m_0101ori-words2: “[...] For the purposes of section 18(1) of the Act, trade marks consisting of words in Mãor will be treated in the same way as trade marks consisting of the equivalent English words." 
Adicionalmente, toda vez que el gentilicio deriva generalmente del nombre con el que se conoce a una determinada comunidad indígena, compartiendo por tanto la misma raíz gramatical, puede resultar comprensible que la protección -otorgada por la legislación interna correspondiente- se extienda incluso al gentilicio.

En Canadá, por ejemplo, las comunidades indígenas gozan de esta protección mínima gracias a una disposición particular. En efecto, la sección 9 de la Ley Canadiense de Marcas ("Canadian Trademarks Act”) prohíbe la adopción, en conexión con un negocio, de placas, escudos, emblemas o marcas que hayan sido adoptados y usados por una "autoridad pública" ("public authority") ", concepto que comprende a las organizaciones o asociaciones indígenas que cumplen ciertos requisitos exigidos por la ley canadiense ${ }^{10}$.

En consecuencia, diversas comunidades indígenas canadienses han protegido sus propios nombres. Por ejemplo, el nombre "SKATIN" en favor del pueblo indígena Skatin Nations, "KASKA" en favor del Kaska Tribal Council, "QUENEESH" en favor de Comox Band of Indians, "NK'MIP" en favor de Osoyoos Indian Band y "FIRST NATIONS SUMMIT" en favor de First Nations Summit ${ }^{11}$. Así, por ejemplo, es posible citar la exitosa oposición presentada por la Asamblea del pueblo indígena First Nations en contra de la solicitud de registro del signo "FIRST NATION", presentada por una empresa de Winnipeg ${ }^{12}$.

Es posible citar un célebre caso acontecido en Estados Unidos de América (EEUU), conocido como "Navajo Nation v. Urban Outfitters", donde la multinacional estadounidense Urban Outfitters decidió comercializar productos con los nombres "NAVAJO" y "NAVAHO" y reproducir los diseños tradicionales de la Nación Navajo ("Navajo Nation") sin la debida autorización, con la finalidad de aprovecharse de la reputación ganada por la calidad y diversidad de sus artes (bisutería, cerámica, tapices y pinturas). La Nación Navajo decidió recurrir a los tribunales por ser titular de 86 marcas registradas ante la USPTO y haber usado el nombre "NAVAJO" en el comercio desde 1894. En septiembre de 2016, ambas partes finalmente llegaron a un acuerdo, en virtud del cual se obligaron a comercializar conjuntamente productos "NAVAJO" auténticos (Albino, 2017).

En el ámbito de la CAN, contamos con el primer supuesto contenido en la causal de irregistrabilidad contemplada en el literal g) del artículo 136 de la Decisión 486, el cual establece la irregistrabilidad de signos que consistan en el nombre de comunidades indígenas. Al respecto, en la interpretación prejudicial recaída en el Proceso 187-IP-2015 de 12 de

Trade-marks Act (1985). Prohibited marks9 (1).

10 Boughton Law, "Protecting Aboriginal Marks", julio de 2013, https://www.boughtonlaw.com/2013/07/protectingaboriginal-marks/.

11 Boughton Law, "Protecting Aboriginal Marks".

12 Department of Indian Affairs and Northern Development - Government of Canada, "Intellectual Property and Aboriginal People: A Working Paper" (Minister of Indian Affairs and Northern Development: Ottawa, 1999): 22, https://www.wipo.int/export/sites/www/tk/en/databases/creative_heritage/docs/ip_aboriginal_people.pdf. 
noviembre de 2015, el TJCA manifestó que: "La presente causal de irregistrabilidad se refiere a los nombres de las propias comunidades, como por ejemplo yanomani, aymara o muisca" ${ }^{13}$.

Adicionalmente, en aras de evitar que el "juez nacional" interprete irrestrictamente este primer supuesto de irregistrabilidad, ${ }^{14}$ el TJCA expresó claramente, en el pronunciamiento segundo de la precitada interpretación prejudicial, lo siguiente: "Las dos primeras causales de irregistrabilidad no revisten mayor dificultad debido a que es posible, y además correcto, realizar una interpretación literal." (Subrayado agregado).

De esta manera, el TJCA estableció claramente que el método de interpretación jurídica a ser aplicado, en relación con los dos primeros supuestos comprendidos en la causal de irregistrabilidad contemplada en el inciso g) del artículo 136 de la Decisión 486, debe ser el método literal.

En suma, en lo concerniente al primer supuesto contenido en la causal de irregistrabilidad del literal g) del artículo 136 de la Decisión 486, es decir, aquel referido al propio nombre de las comunidades indígenas, afroamericanas o locales, al igual que en relación con el segundo supuesto contemplado en dicho literal, el TJCA habría optado por confiar en la claridad del enunciado de la norma ("In claris non fit interpretatio"), prefiriendo enfocarse en el tercer supuesto contemplado en la misma, vale decir, en aquel relativo a la irregistrabilidad de signos que constituyan expresión de la cultura o práctica de comunidades indígenas, afroamericanas o locales.

En efecto, al interpretar de manera literal la disposición normativa objeto de análisis, se entiende está referida al nombre mediante el cual es conocida la propia comunidad (e.g., "yanomani”, "aymara" o "muisca"). En tal virtud, el TJCA consideró suficiente advertir, en el pronunciamiento segundo de la interpretación prejudicial recaída en el Proceso 187-IP2015, respecto de la necesidad de emplear el método de interpretación literal o gramatical al aplicar los dos primeros supuestos de la norma comentada.

Evidentemente, el hecho de que el enunciado de la norma comunitaria emplee en su redacción el número singular ("el nombre") y no el plural supone que la protección deba recaer únicamente sobre "el" nombre con el cual es conocida una determinada comunidad indígena, protección que incluso podría extenderse al gentilicio (e.g., "yanomani”, "aymara" o "muisca") pero lógicamente no a otros nombres o denominaciones indígenas que no compartan la misma raíz gramatical.

Sin embargo, en la Sentencia 2007-00012-00, el Consejo de Estado de Colombia equiparó el nombre propio de un personaje histórico indígena (Tisquesusa) con "el" nombre de una comunidad indígena (muisca o chibcha), cuando en realidad estos no comparten siquiera la misma raíz gramatical; contraviniendo de esta manera las directrices interpretativas señaladas

13 Interpretación prejudicial recaída en el Proceso 187-IP-2015 de 12 de noviembre de 2015, párr. 22.

14 En relación con el concepto de "juez nacional”, véase Indacochea (2018). 
por el TJCA, al contrariar el imperativo de interpretar de manera literal los dos primeros supuestos comprendidos en la causal de irregistrabilidad establecida en el literal g) del artículo 136 de la Decisión 486.

En efecto, como se desprende del párrafo 43 de la precitada sentencia, el Consejo de Estado de Colombia habría cometido el desacierto de considerar que tanto el nombre de un personaje histórico indígena, como es el último zipa o líder muisca (Tisquesusa), así como la imagen de una pieza artesanal que forma parte del patrimonio de la nación colombiana (Poporo Quimbaya), constituyen elementos que determinarían que el signo que los comprenda vulnere los tres supuestos contenidos en la causal de irregistrabilidad contemplada en el literal g) del artículo 136 de la Decisión 486.

\section{Irregistrabilidad de signos utilizados para distinguir productos de comunidades indígenas}

En relación con el segundo supuesto contenido en el literal g) del artículo 136 de la Decisión 486, vale decir, aquel referido a la irregistrabilidad de signos utilizados para distinguir productos -así como la forma de procesarlos- o servicios de comunidades indígenas, afroamericanas o locales, es pertinente citar la interpretación prejudicial recaída en el emblemático Proceso 60-IP-2012 $2^{15}$.

En dicha interpretación prejudicial, el TJCA explicó claramente que la finalidad de la prohibición contenida en el segundo supuesto de la causal establecida en el literal g) del artículo 136 es evitar que el uso de determinados signos sugiera una falsa relación entre el producto o servicio y la comunidad indígena. En otras palabras, el objetivo de este segundo supuesto es a fin de cuentas, evitar el riesgo de confusión en el público consumidor, como se desprende del párrafo transcrito a continuación:

"El objetivo es que el uso de dichos símbolos o signos no sugiera una falsa relación entre el producto y/o servicio y la comunidad indígena, ya que el consumidor propenderá a atribuirle a los productos y/o servicios identificados, las cualidades y las características inherentes a la imagen de la comunidad indígena aludida, y aún en el caso en que no atribuya una específica calidad, igualmente relacionará el producto y/o servicio con la comunidad, creyendo erróneamente que le pertenece la fabricación y la producción del mismo o que, en todo caso, se encuentra relacionado a la comunidad productora"16. (Subrayado agregado al texto).

\footnotetext{
15 Interpretación prejudicial recaída en el Proceso 60-IP-2015, 24 de octubre de 2012, intranet.comunidadandina.org/documentos/Procesos/60-IP-2012.doc.

16 Interpretación prejudicial recaída en el Proceso 60-IP-2012, 20, acápite 6.
} 
En consecuencia, el segundo supuesto comprendido en la causal de irregistrablidad establecida en el literal g) del artículo 136 de la Decisión 486 se encuentra dirigido a impedir que el consumidor relacione de cualquier manera un determinado producto o servicio con una comunidad en particular, sin que éstos realmente se encuentren relacionados, y así evitar que se produzca un riesgo de confusión. De esta manera, el objetivo perseguido por este segundo supuesto contenido en la causal de irregistrabilidad establecida en el literal g) del artículo 136 de la Decisión 486 -al igual que en todos los casos mencionados al final del presente acápite- sería evitar que el consumidor sea inducido a error.

Como explica Jumadla, una marca es, ante todo, un indicador de origen que permite distinguir los productos de un fabricante o vendedor de los de otro. En ese sentido, el uso de nombres, marcas o símbolos indígenas probablemente pueda sugerir una conexión entre los productos que llevan estos nombres, marcas o símbolos y los pueblos o comunidades indígenas que lo han creado y usado, y que precisamente han venido siendo conocidos gracias a estos nombres, marcas o símbolos durante varias generaciones; especialmente, cuando la comunidad indígena elabora productos idénticos o similares, ofrece servicios idénticos o similares, o se dedica a un negocio idéntico o similar al del tercero apropiador. La asociación engañosa sería perjudicial no solo para la comunidad indígena, sino también para el público consumidor en general (Jumadla, 2010).

Adicionalmente, el TJCA señaló que los productos artesanales y la manera de procesarlos (i.e., elaborarlos, fabricarlos, producirlos, etc.) suponen la aplicación de conocimientos tradicionales: ${ }^{17}$

"El Tribunal considera que los productos artesanales y el procesamiento de especies para hacer alimentos entran dentro del concepto de "conocimientos tradicionales", ya que dicho procesamiento y elaboración de productos cuentan con una determinada carga cultural y arraigo histórico"18 .

El TJCA arribó a esta conclusión luego de citar una publicación de la Comunidad Andina (CAN) en la cual se proveían elementos para la protección sui generis de los conocimientos tradicionales colectivos e integrales desde la perspectiva indígena. Igualmente, resulta apropiado, transcribir una definición amplia, realizada por la $\mathrm{OMPI}^{19}$, del concepto de "conocimientos tradicionales":

"La OMPI utiliza actualmente el término "conocimientos tradicionales" para referirse a las obras literarias, artísticas o científicas basadas en la

17 En relación con los conocimientos tradicionales, cabe mencionar que en el pie de página 17, párr. 12, de la interpretación prejudicial recaída en el Proceso 187-IP-2015, se cita al autor de estas líneas, Indacochea (2014: 175176).

18 Interpretación prejudicial recaída en el Proceso 60-IP-2012, 25.

19 OMPI, "Conocimientos tradicionales: necesidades y expectativas en materia de propiedad intelectual. Informe relativo a las misiones exploratorias sobre propiedad intelectual y conocimientos tradicionales (1998-1999)", 2001, 25-26, https://www.wipo.int/edocs/pubdocs/es/tk/768/wipo_pub_768.pdf. 
tradición; así como las interpretaciones o ejecuciones; invenciones; descubrimientos científicos; dibujos o modelos; marcas, nombres y símbolos; información no divulgada y todas las demás innovaciones y creaciones basadas en la tradición que proceden de la actividad intelectual en el ámbito industrial, científico, literario o artístico. La expresión "basadas en la tradición" se refiere a los sistemas de conocimiento, creaciones, innovaciones y expresiones culturales que: se han transmitido generalmente de generación en generación; se considera generalmente que pertenecen a un pueblo en particular o a su territorio, y evolucionan constantemente en respuesta a los cambios que se producen en su entorno. Entre las categorías de conocimientos tradicionales figuran: los conocimientos agrícolas; los conocimientos científicos; los conocimientos técnicos; los conocimientos ecológicos; los conocimientos medicinales, incluidos las medicinas y los remedios conexos; los conocimientos relacionados con la diversidad biológica; las "expresiones del folclore" en forma de música, baile, canción, artesanía, dibujos y modelos, historias y obras de arte; elementos de los idiomas, como los nombres, indicaciones geográficas y símbolos, y bienes culturales muebles. Quedarían excluidos de esta descripción de los conocimientos tradicionales los elementos que no se derivan de la actividad intelectual en el ámbito industrial, científico, literario o artístico, como los restos humanos, los idiomas en general y otros elementos similares del "patrimonio" en un sentido amplio." (Subrayado agregado al texto).

De esta definición amplia de "conocimientos tradicionales" es posible diferenciar con suficiente precisión lo que formaría parte de este concepto de aquello que no, por cuanto se trata de una definición positiva y, a la vez, de una negativa. En efecto, se desprende del texto transcrito que los bienes culturales muebles (e.g., Poporo Quimbaya) se encontrarían comprendidos dentro del concepto -en sentido amplio- de "conocimientos tradicionales". Sin embargo, "quedarían excluidos [...] los elementos que no se derivan de la actividad intelectual en el ámbito industrial, cientifico, literario o artístico, como los restos humanos, los idiomas en general y otros elementos similares del "patrimonio" en un sentido amplio" (e.g., Tisquesusa o el nombre de un personaje histórico indígena).

A este respecto, conviene aclarar que, si bien todos los conocimientos indígenas son conocimientos tradicionales, no todos los conocimientos tradicionales son conocimientos indígenas; por cuanto, en una relación género-especie, los conocimientos tradicionales son el género, mientras que los conocimientos indígenas constituyen la especie. En efecto, si bien las comunidades indígenas son las más representativas por su interacción con estos conocimientos, también las comunidades locales son tenedoras de conocimientos tradicionales (Núñez, 2018: 18; Brush, 2005: 99-100). En tal virtud, cobra sentido el haber incluido a las comunidades locales en el enunciado del literal g) del artículo 136 de la Decisión 486. 
Adicionalmente, es preciso explicar que las causales de irregistrabilidad relacionadas con pueblos o comunidades indígenas varían de legislación en legislación, existiendo una obligación a nivel multilateral -de los países miembros de los correspondientes acuerdosúnicamente en cuanto a la necesidad de impedir el registro de signos (así como de patentes de invención o certificados de obtentor de variedad vegetal) que supongan una apropiación de conocimientos tradicionales, principalmente en virtud del régimen instaurado por el Convenio sobre la Diversidad Biológica $(\mathrm{CDB})^{20}$; así como de aquellos signos que constituyan expresiones culturales tradicionales, también denominadas expresiones del folclore, en virtud de los diversos tratados promovidos por la Organización de las Naciones Unidas para la Educación (UNESCO) que rigen la materia ${ }^{21}$.

Sin embargo, es necesario indicar, en relación con la irregistrabilidad de signos que constituyan expresiones del folclore, que la mayoría de ordenamientos jurídicos se han abstenido de adoptar tales regímenes. Unos porque han admitido el folclore como objeto de protección por parte de los derechos de autor y derechos conexos. Otros, porque simplemente lo consideran parte del dominio público (Moura, 2009: 197).

Antes de concluir el presente acápite, es preciso comentar que, en el derecho comparado, existe un método o medida de protección denominada "Etiquetas de Autenticidad" ("Labels of Authenticity"), inicialmente empleada en Canadá mediante el registro de la marca "IGLOO", iniciativa que fuera seguida por el "Silver Hand Program" en Alaska 22 , y posteriormente por Nueva Zelanda e incluso Australia (Chalk y Dunlop, 2009: 956-982).

El primer país en registrar este tipo de marcas fue Canadá, cuando en 1958 estableció la marca "IGLOO" para proteger productos de las comunidades Dene, Métis e Inuvialuit (Inuits del ártico occidental) del norte de Canadá. Si bien la presencia del símbolo del "IGLOO" en artesanía tradicional ha logrado aumentar la confianza del consumidor en productos indígenas y proteger el trabajo de los artesanos indígenas canadienses, el éxito y la longevidad de esta marca la han hecho víctima de imitación y parasitismo, ante lo cual se

20 El sistema que tiene como instrumento constitutivo el Convenio sobre Diversidad Biológica (CDB), firmado en Río de Janeiro el 5 de junio de 1992 (EEUU es firmante no ratificado), se encuentra además comprendido por el Protocolo de Cartagena sobre Seguridad de la Biotecnología del Convenio sobre Diversidad Biológica (Protocolo de Cartagena), adoptado el 29 de enero de 2000, y por el Protocolo de Nagoya sobre acceso a los recursos genéticos y participación justa y equitativa en los beneficios derivados de su utilización al Convenio sobre la Diversidad Biológica (Protocolo de Nagoya), adoptado el 29 de octubre de 2010.

21 Entre los principales instrumentos internacionales promovidos por la Organización de las Naciones Unidas para la Educación, la Ciencia y la Cultura (UNESCO), organización competente en la materia, se encuentran la Convención para la Salvaguarda del Patrimonio Cultural Inmaterial, aprobada el 17 de octubre de 2003 y la Convención sobre la protección y la promoción de la diversidad de las expresiones culturales, aprobada el 20 de octubre de 2005 . Véase también, OMPI, "La protección de las expresiones culturales tradicionales/expresiones del folclore: Objetivos y principios revisados", documento WIPO/GRTKF/IC/9/4, de 9 de enero de 2006, https://www.wipo.int/edocs/mdocs/tk/es/wipo_grtkf_ic_9/wipo_grtkf_ic_9_4.doc.

22 Programa administrado por el "Department of Indian Affairs and Northern Development", órgano encargado de otorgar las licencias sobre el uso sobre la marca. 
optó por restringir la impresión o reproducción de la etiqueta correspondiente al "IGLOO" a una sola empresa ${ }^{23}$.

Por su parte, la marca oficial "GENUINE COWICHAN", registrada a favor del Cowichan Band Council, cumple una doble función, como un tipo de marca de certificación certificando el cumplimiento de los estándares establecidos-, y como una indicación geográfica ${ }^{24}$. El Cowichan Band Council también tiene la titularidad de las marcas de certificación "COWICHAN"25 y "GENUINE COWICHAN APPROVED" (Chalk y Dunlop, 2009: 964).

En EEUU, la Ley de Artes y Oficios Indígenas ("Indian Arts and Crafts Act") impide publicitar o comercializar productos como hechos por indígenas ("Indian made") cuando éstos no hayan sido realmente elaborados por artesanos indígenas ${ }^{26}$; estableciendo que es un delito vender productos de modo que se sugiera falsamente que son producidos por indígenas. Asimismo, según la citada ley, las comunidades indígenas afectadas pueden pedir indemnización y/o medidas cautelares, además del retiro de los productos engañosos del mercado ${ }^{27}$.

Adicionalmente, el Consejo de Agricultura Intertribal ("Intertribal Agriculture Council") de EEUU ha otorgado más de 500 licencias renovables cada año sobre el uso de su marca "MADE BY AMERICAN INDIANS®", registrada en 1995 por la USPTO para la promoción de productos agrícolas u otros productos obtenidos o tratados por miembros inscritos de comunidades indígenas reconocidas (Dutfield, 1999: 57).

Finalmente, cabe mencionar que, en Nueva Zelanda, se han establecido -y popularizadouna serie de marcas para garantizar la procedencia de arte, artesanía y performances maoríes. De esta manera, existen tres clases de protección ("TOI IHO"): (i) la marca "MAORI", reservada para artistas descendientes de maoríes; (ii) la marca "MAINLY MAORI", para grupos de artistas mayoritariamente maoríes; y, (iii) la marca "MAORI COPRODUCTION", que reconoce la cotitularidad entre artistas maoríes y no-maoríes (Chalk y Dunlop, 2009. ${ }^{28}$

En Australia, en cambio, si bien se implementó temporalmente un método de protección similar al empleado en Nueva Zelanda mediante un registro administrado por la "National

23 Arts+Law, "Trade mark protection and ICIP: How does Australia fare?", 31 de diciembre de 2006, 31 de diciembre de 2006, https://www.artslaw.com.au/articles/entry/trade-mark-protection-and-icip-how-does-australia-fare/.

24 Boughton Law, "Protecting Aboriginal Marks".

25 Canadian Trademark no. 465836.

26 WIPO, "Intellectual Property and Traditional Cultural Expressions/Folklore", WIPO Publication no. 913(E), http://pmg-assets.s3-website-eu-west-1.amazonaws.com/docs/110817wipo1_0.pdf.

27 APO, "New tracks: Indigenous knowledge and cultural expression and the Australian intellectual property system", 31 de mayo de 2012, 20, http://apo.org.au/system/files/38273/apo-nid38273-68691.pdf.

28 Véase también, Arts+Law, "Trade mark protection and ICIP: How does Australia fare?”; y, WIPO, "Traditional Knowledge and Intellectual Property - Background Brief” https://www.wipo.int/pressroom/en/briefs/tk_ip.html. "Trademarks can be used to identify authentic indigenous arts, as the Maori Arts Board in New Zealand, Te Waka Toi, has done. Some countries also have special legislation for the protection of folklore. Panama has established a registration system for traditional cultural expressions, while the Pacific Regional Framework. for the Protection of Traditional Knowledge and Expressions of Culture gives "traditional owners" the right to authorize or prevent use of protected folklore and receive a share of the benefits from any commercial exploitation." 
Indigenous Arts Advocacy Association" (NIAAA), éste no habría sido tan popular como en Nueva Zelanda. En consecuencia, al no encontrarse más operativa la NIAAA, las marcas protegidas por ella no fueron renovadas y, por consiguiente, fueron removidas del registro de marcas australiano en 2009 (Chalk y Dunlop, 2009: 968).

\section{Irregistrabilidad de signos que constituyan la expresión de la cultura o práctica de comunidades indígenas}

En relación con este último supuesto, es preciso aclarar que no cualquier tipo de apropiación cultural a través de un registro marcario es sancionada con la irregistrabilidad o, de ser el caso, con la nulidad del correspondiente registro, sino únicamente la apropiación de expresiones culturales tradicionales o del folclore. Adicionalmente, diversos instrumentos de la UNESCO vinculantes en la materia establecen un requisito esencial para la protección de tales expresiones, a saber, que sean "resultantes de la creatividad humana".

En efecto, en lo concerniente al último supuesto contenido en la causal de irregistrabilidad del literal g) del artículo 136 de la Decisión 486, referido a signos que constituyan expresiones culturales tradicionales (i.e., expresiones del folclore) de comunidades indígenas, afroamericanas o locales, el TJCA explicó, con ocasión de la interpretación prejudicial pronunciada en el marco del Proceso 187-IP-2015, que una característica sine qua non de toda expresión cultural es la de ser producto de la creatividad humana.

De esta manera, el TJCA manifestó, en el párrafo 33 de la interpretación prejudicial recaída en el Proceso 187-IP-2015, siguiendo una interpretación sistemática sobre la base de un análisis comparado de los diferentes instrumentos internacionales que rigen la materia, lo siguiente:

"33. De esta manera, la noción de "expresión del folclore" alude a creaciones artísticas, generalmente colectivas, que reflejen expectativas artísticas tradicionales. Ellas pueden ser verbales, musicales, corporales o tangibles. Por consiguiente, un cuento (expresión verbal), una canción (expresión musical), una danza (expresión corporal) o una cerámica (expresión tangible) inspiradas en un personaje histórico indígena, podrían ser consideradas como producciones integradas por elementos del patrimonio artístico tradicional, resultantes de la creatividad de personas o grupos con contenido cultural (Convención sobre la protección y la promoción de la diversidad de las expresiones culturales), que además reflejan expectativas artísticas tradicionales." (Subrayado comprendido en el texto original). 
En consecuencia, el TJCA equipara los conceptos de "expresión de la cultura o práctica" con aquellos de "expresión cultural tradicional" y "expresión del folclore", describiendo asimismo cuatro características propias de estas expresiones:

(i) aluden a "creaciones artísticas";

(ii) reflejan "expectativas artísticas tradicionales";

(iii) pueden considerarse como producciones integradas por elementos del "patrimonio artístico tradicional"; y,

(iv) son "resultantes de la creatividad" humana.

$\mathrm{Al}$ analizar estas características, es posible inferir que tales expresiones del folclore o expresiones culturales tradicionales tienen siempre un componente esencial de "creatividad artística"; condición necesaria que es reiterada en los párrafos posteriores. De esta manera, en el párrafo 39 de la interpretación prejudicial pronunciada en el marco del Proceso 187-IP2015, el TJCA indicó lo siguiente:

"[...] lo establecido en cuanto a signos que "constituyan la expresión de su cultura o práctica" se refiere a expresiones artísticas, sean éstas verbales, musicales, corporales o tangibles. [...] Siendo posible apreciar que la mencionada figura protege las distintas expresiones resultantes de la creatividad humana." (Subrayado agregado al texto).

Como es posible apreciar, el TJCA estableció expresamente que la norma se refiere necesariamente a "expresiones artísticas", las cuales lógicamente deben ser "resultantes de la creatividad humana". De lo anterior, es posible extraer las siguientes dos condiciones necesarias y esenciales (condiciones sine qua non) para que se configure el tercer supuesto de irregistrabilidad contemplado en la norma:

(i) Que se trate de "expresiones artísticas"; y,

(ii) Que sean "resultantes de la creatividad humana".

Adicionalmente, el TJCA mencionó, en el párrafo 39 de la interpretación prejudicial objeto de estudio, las diferentes formas de expresiones artísticas o culturales susceptibles de ser protegidas (verbales, musicales, corporales o tangibles), brindando además ejemplos de cada una. Asimismo, el TJCA destacó que todas esas expresiones artísticas podrían ser "inspiradas" en un personaje histórico indígena, poniendo énfasis en la necesidad de que sean "resultantes de la creatividad humana", lo cual es reiterado en el párrafo 40 de la interpretación prejudicial comentada, párrafo que es a su vez replicado en el pronunciamiento tercero:

"40. En consecuencia, un cuento (expresión verbal), una canción (expresión musical), una danza (expresión corporal) o una cerámica 
(expresión tangible) inspiradas en un personaje histórico indígena, podrían ser consideradas como producciones integradas por elementos del patrimonio artístico tradicional, resultantes de la creatividad de personas o grupos con contenido cultural (Convención sobre la protección y la promoción de la diversidad de las expresiones culturales), que además reflejan expectativas artísticas tradicionales." (Subrayado agregado al texto).

En consecuencia, el TJCA establece como condición esencial para la protección de una determinada expresión de la cultura o práctica de comunidades indígenas, afroamericanas o locales -y su consiguiente irregistrabilidad- que éstas sean "resultantes de la creatividad bumana" o, más precisamente, "resultantes de la creatividad de personas o grupos con contenido cultural [...], que además reflejan expectativas artísticas tradicionales".

A efectos de determinar si la marca registrada en el caso concreto se encontraría incursa en el último supuesto contenido en la causal contemplada en el literal g) del artículo 136 de la Decisión 486, siguiendo los criterios interpretativos indicados por el propio TJCA, habría correspondido analizar en el proceso interno si el nombre "Tisquesusa" constituía propiamente una expresión "resultante de la creatividad humana", en cuyo caso sería irregistrable como marca, o si, por el contrario, se trataría del nombre de un personaje histórico real -en contraposición con uno "resultante de la creatividad humana"-, en cuyo caso no existiría ningún impedimento para su registro como marca.

Si bien, en la Sentencia 2007-00012-00, el propio Consejo de Estado afirmó que "Tisquesusa" es considerado el último Zipa de la comunidad Muisca, contradictoriamente concluyó que la marca otorgada debía ser anulada toda vez que el nombre "Tisquesusa" hace parte del patrimonio cultural de la nación colombiana, afirmación que revelaría un erróneo entendimiento de la interpretación prejudicial, por cuanto el objeto de protección no sería todo tipo de patrimonio cultural sino únicamente el "patrimonio artístico tradicional", tal como se señala, tanto en el párrafo 33 como en el 40, así como en el pronunciamiento tercero, de la interpretación prejudicial recaída en el Proceso 187-IP-2015: "[...] producciones integradas por elementos del patrimonio artístico tradicional, resultantes de la creatividad de personas o grupos con contenido cultural [...]'.

En otras palabras, el estatus de patrimonio cultural no debería incidir en la calificación de una determinada expresión como "expresión de la cultura o práctica" (i.e., expresión tradicional o del folclore) de comunidades indígenas, afroamericanas o locales; por cuanto la disposición comentada no protegería todo patrimonio cultural, sino únicamente el "patrimonio artístico tradicional"; es decir, aquel "resultante de la creatividad humana".

Adicionalmente, cabe destacar que, en la mayoría de casos, los nombres de personajes históricos se encontrarían ya dentro de la esfera del dominio público, como lo evidencia la existencia de numerosas marcas de servicios o nombres comerciales, en rubros como la hotelería o la restauración, que contienen nombres de personajes históricos. Por ejemplo, 
en relación con los libertadores Simón Bolívar y José de San Martín, es posible citar los siguientes nombres comerciales o establecimientos ubicados en el continente americano: Café Bolívar (1741 Ocean Park Blvd, Santa Mónica, CA. 90405, EEUU), Bolívar Restaurant Bar Lounge (841 Washington Ave Miami Beach FL 33139, EEUU), Gran Hotel Bolívar (Jirón de La Unión 958, Lima, Perú), Hotel Bolívar Plaza Pasto (Cra. 7 \# 21-21, Pasto, Nariño, Colombia), Hotel Bolívar (Av. Demetrio Mendoza Vía A San Luis, Cúcuta, Norte de Santander, Colombia), Hotel Bolívar Plaza (Calle 21 \# 14-17, Armenia, Quindío, Colombia), Hotel Bolívar (Bolívar 886, San Telmo, 1066 Buenos Aires, Argentina), Hotel Bolívar Plaza (Calle 22 n²2-40, Manizales, Caldas, Colombia), Hotel Bolívar (Santa Bárbara de Zulia 5148, Zulia, Venezuela); Café San Martin (112 N A St, Lompoc, CA 93436, EEUU), panaderías-café San Martín (Guatemala y El Salvador), Hotel San Martín (San Martín 667, Viña del Mar 2520000, Valparaíso, Chile), Café San Martín (Gral. San Martín 501-555, Cipolletti, Río Negro, Argentina), Café San Martín (Córdoba 24, Oberá, Misiónes, Argentina), Hotel San Martín (Santa Fe 955/73, 3400 Corrientes, Argentina), Hotel San Martín (Alsina y Pellegrini, Santa Rosa, La Pampa, Patagonia, Argentina), Hotel San Martín (G. Espejo 435, M5502AVL Mendoza, Argentina) y Hotel San Martín (Av. Callao 327, 1022 Buenos Aires, Argentina).

De igual manera, existen innumerables nombres de personajes históricos indígenas comúnmente usados para distinguir servicios de hotelería y restauración en la subregión andina, entre los cuales se encuentran: Hotel Museo Manco Capac (Av. Manco Capac 420, Cusco, Cusco, Peru), Posada Inca Manco Capac (Av. Manco Capac 617 A, Wanchaq, Cusco, Perú), Hotel Manco Capac (Jr. Manco Manco Capac 575, Jauja, Perú), Hostal Manco Capac (Av. Manco Capac 108, Baños del Inca, Cajamarca, Perú), Hotel Manco Capac Inn (Jirón Tacna, 277, Puno, Perú), Hotel Pachakuteq (Avenida Pachacutec, 809, Aguas Calientes, Machu Picchu, Cusco, Perú), Pachacuteq Hostel (Av. La Paz C26, Cusco, Cusco, Perú), Pachacutec Grill \& Bar (Portal de Panes 105, Cusco, Cusco, Perú), Hostal Pachacuteq Inn (B-3-A, Cusco, Cusco, Perú), Hotel Pachacutec Cusco Inn (C 13, Av. Infancia, Cusco, Perú), bar-restaurante Sisa Atahualpa (Carrera 10 oeste \# 1-15 esquina, Cali), Restaurant Atahualpa (Pj. Atahualpa 686 Br. Cajamarca, Perú), Hotel Atahualpa (Mariscal Sucre 3-50, Cuenca, Ecuador), Café Lautaro (Av. San Martín 96, 9420 Río Grande, Tierra del Fuego, Argentina), Hotel Boutique Lautaro (Erasmo Escala 400, Lautaro, Araucania, Chile), Hotel Colonial Lautaro (Barros Arana 1105, Lautaro, Araucania, Chile), Café Tisquesusa (Panamericana, Manizales, Colombia) y Hotel Tisquesusa (Calle $18 \mathrm{n}^{\circ}$ 7-43, Girardot, Colombia).

En suma, siguiendo las directrices interpretativas establecidas por el TJCA, a efectos de determinar si la marca "Tisquesusa" se encontraba incursa en el último supuesto de irregistrabilidad analizado, habría correspondido evaluar en el proceso interno si dicho nombre constituía propiamente una expresión "resultante de la creatividad humana" -en cuyo caso sería irregistrable como marca-, o si, por el contrario, se trataría del nombre de un personaje histórico real -en cuyo caso no existiría ningún impedimento para su registro como marca-. 
Resulta contradictorio - si es que no imposible- que el nombre de un personaje histórico indígena sea "resultante de la creatividad humana", por cuanto no podría tratarse de un personaje histórico real y, a su vez, de uno ficticio, producto del ingenio humano. En consecuencia, en lugar de pretender subsumir el presente caso en la tercera hipótesis contemplada en el literal g) del artículo 136 de la Decisión 486, probablemente hubiera sido más apropiado declarar la nulidad de la resolución impugnada únicamente sobre la base del segundo supuesto comprendido en la referida disposición normativa.

\section{Conclusiones}

En la Sentencia 2007-00012-00, el Consejo de Estado concluyó que la inclusión, en el signo solicitado y posteriormente registrado, del término "Tisquesusa" y del elemento gráfico que reproduce el denominado "Poporo Quimbaya", vulnerarían los tres supuestos de la causal de irregistrabilidad contemplada en el literal g) del artículo 136 de la Decisión 486.

Sin embargo, como se explicó anteriormente, no es correcto afirmar que el nombre de un personaje histórico indígena constituya "el" nombre de una comunidad indígena, en virtud de lo establecido por el TJCA en cuanto al imperativo de interpretar de manera literal -y por consiguiente restrictiva- los dos primeros supuestos de irregistrabilidad contenidos en el literal g) del artículo 136 de la Decisión 486.

De igual manera, resulta erróneo considerar el nombre de un personaje histórico indígena como "expresión de [la] cultura o práctica" (i.e., expresión cultural tradicional o del folclore) de una determinada comunidad indígena, por cuanto no cumpliría con la condición sine qua non, establecida en diversos instrumentos de la UNESCO y recogida por el TJCA: ser una expresión artística "resultante de la creatividad bumana".

En consecuencia, probablemente hubiera sido más conveniente para el Consejo de Estado, a fin de no caer en imprecisiones en la aplicación del ordenamiento jurídico comunitario andino o, en todo caso, de evitar contrariar las directrices interpretativas establecidas en la interpretación prejudicial pronunciada en el marco del mismo proceso, declarar la nulidad de la Resolución n 8885 de 11 de abril de 2006 únicamente sobre la base del segundo supuesto comprendido en la causal de irregistrabilidad contemplada en el literal g) del artículo 136 de la Decisión 486.

Si bien parecería cuestionable la decisión del Consejo de Estado de declarar la irregistrabilidad de la marca de servicio "Tisquesusa" por vulnerar todos los supuestos contenidos en la causal de irregistrabilidad contemplada en el literal g) del artículo 136 de la Decisión 486, resulta innegable que el máximo tribunal colombiano fundamentó debidamente la contravención respecto del segundo supuesto comprendido en el citado literal, toda vez que el uso del signo 
solicitado para distinguir "servicios de restauración (alimentación); hospedaje temporal, cafés, bares, restaurantes, grilles y similares" puede efectivamente llevar al público consumidor a creer erróneamente que los productos brindados en el marco de la prestación de tales servicios son obtenidos o elaborados por miembros de la comunidad indígena muisca.

\section{Referencias bibliográficas}

ALBINO, Vera (2017). "The right of Indigenous People to protect their Intellectual Property", en Inventa International, 25 de octubre de 2017, https://inventa.com/en/news/article/267/the-right-ofindigenous-people-to-protect-their-intellectual-property.

BRUSH, Stephen B. (2005). "Protecting Traditional Agricultural Knowledge", Washington University Journal of Law \& Policy, 17.

CHALK, Peter J. y Alexander Dunlop (2009). "Indigenous Trade Marks and Human Rights: An Australian and New Zealand Perspective", The Trademark Reporter (The Law Journal of the INTA), 99 (4): 956-982, https://www.inta.org/TMR/Documents/Volume\%2099/vol99_no4_a3.pdf.

DEPARTMENT OF INDIAN AFFAIRS AND NORTHERN DEVELOPMENT, Government of Canada (1999). "Intellectual Property and Aboriginal People: A Working Paper" (Minister of Indian Affairs and Northern Development: Ottawa), https://www.wipo.int/export/sites/www/tk/en/databases/creative_heritage/docs/ip_aboriginal_ people.pdf.

DUTFIELD, Graham (1999). “Intellectual Property Rights, Trade and Biodiversity: Seeds and Plant Varieties", Montreal, https://pdfs.semanticscholar.org/c385/5011ecb2e1bb8ecb8fcecf660a8abab55ca7.pdf.

INDACOCHEA, Juan Manuel (2014). "Derechos de Propiedad Intelectual Tradicional. La nueva generación de derechos de Propiedad Intelectual”, Ius Inter Gentes, 10 (10): 166-194.

INDACOCHEA, Juan Manuel (2018), "La interpretación prejudicial 242-IP-2015 del Tribunal de Justicia de la Comunidad Andina”, Revista Tribuna Internacional, 7 (13), https://tribunainternacional.uchile.cl/index.php/RTI/article/view/49647/53304.

IPONZ, "Absolute grounds distinctiveness. Section 18 of the Trade Marks Act 2002", https://www.iponz.govt.nz/about-ip/trade-marks/practice-guidelines/current/absolute-groundsdistinctiveness/\#jumpto-6__002e3-m__0101 ori-words2.

JUMADLA, Anne Marie Celeste (2010), "Philippines: Indigenous Names, Marks and Symbols", http://sapalovelez.com/v2/wp-content/uploads/2016/01/Philippines_-Indigenous-names-marksand-symbols.pdf. 
Revista Tribuna Internacional

Volumen $8 \cdot \mathrm{N}^{\circ} 15 \cdot 2019$

ISSN 0719-482X (versión en línea)

MOURA V., Dário (2009). "La propriété intellectuelle en droit international privé" (Académie de Droit International de La Haye: La Haya).

NÚÑEZ D., Solangs (2018). "Los conocimientos tradicionales como materia de la propiedad intelectual” (Bogotá: Leyer Editores).

OMPI (2001), "Conocimientos tradicionales: necesidades y expectativas en materia de propiedad intelectual. Informe relativo a las misiones exploratorias sobre propiedad intelectual y conocimientos tradicionales (1998-1999)", https://www.wipo.int/edocs/pubdocs/es/tk/768/wipo_pub_768.pdf.

OMPI (2006), "La protección de las expresiones culturales tradicionales/expresiones del folclore: Objetivos y principios revisados", documento WIPO/GRTKF/IC/9/4, https://www.wipo.int/edocs/mdocs/tk/es/wipo_grtkf_ic_9/wipo_grtkf_ic_9_4.doc.

WIPO, "Intellectual Property and Traditional Cultural Expressions/Folklore", WIPO Publication No 913(E), http://pmg-assets.s3-website-eu-west-1.amazonaws.com/docs/110817wipo1_0.pdf.

WIPO, “Traditional Knowledge and Intellectual Property - Background Brief”, https://www.wipo.int/pressroom/en/briefs/tk_ip.html. 